\title{
Acute effects of isoinertial resistance application on sprint, vertical and horizontal jump performance
}

\author{
Kale M. ${ }^{1 \mathrm{ABCDE}}$, Özmusul A. ${ }^{2 \mathrm{BCDE}}$, Heper E. ${ }^{1 \mathrm{ABDE}}$ \\ ${ }^{1}$ Department of Coaching Education, Faculty of Sport Sciences, Eskişehir Technical University, Eskişehir, Turkey \\ ${ }^{2}$ Department of Physical Education and Sports, Graduate School of Health Sciences, Anadolu University, Eskişehir,
} Turkey.

Authors' Contribution: A - Study design; B - Data collection; C - Statistical analysis; D - Manuscript Preparation; E - Funds Collection.

\begin{abstract}
Purpose: The purpose of this study was to investigate of the acute effects of isoinertial resistance application on sprint, vertical and horizontal jump performance.

Material: $\quad$ Volunteered 32 male students were divided into randomized experimental group and control group. Before and after 4-repetition maximal back squat with (for experimental group) or without isoinertial resistance application (for control group), $30 \mathrm{~m}$ sprint, vertical squat and countermovement jump tests with both legs, dominant leg, and non-dominant leg, standing broad jump and standing broad countermovement jump tests with both legs were assessed. Wilcoxon t test for pre- and post-test differences within the group, and Mann Whitney $U$ test for pre- and post-test differences between the groups were used.

Results: $\quad$ There were no significant differences within the group and between the groups for $30 \mathrm{~m}$ both legs sprint parameters except pre-test mean stride length between the groups $(Z=-2.27 ; p<0.02)$. Control group had a significant difference between pre- and post-test dominant leg vertical force $(Z=-2.07 ; p<0.05)$. There were significant group differences in pre-test non-dominant leg countermovement jump $(Z=-2.13 ; p<0.05)$, preand post-test non-dominant leg stride length $(Z=-1.99$ and $Z=-2.28 ; p<0.05$, respectively).

Conclusions: As a result of the effect of acute isoinertial resistance application to stride length especially to non-dominant stride length it is concluded that long-term isoinertial resistance training can support sprint performance with tolerating stride length imbalance between dominant leg and non-dominant leg.

Keywords: isoinertial resistance, stride length, vertical jump, horizontal jump, dominant leg, non-dominant leg.
\end{abstract}

\section{Introduction}

Since force and force production of neuromuscular system is critically important for high level performance in each sport event, most appropriate training methods are determined to develop power [1]. Isoinertial eccentric training system that develops motor coordination based on proprioception, balance, neuromuscular efficiency and posture control by developing neuromuscular activity and that becomes one of the popular training methods is a technology that reveals equal amount of eccentric force against concentric force (isoinertial) applied by athletes [2]. Since it is short and high intensity training, this training will increase power of athletes by enabling force and speed in muscle activities [3] and compared to weight training in eccentric muscle contraction, it provides higher muscle activation [4]. Therefore, it was determined that isoinertial strength, speed, and force relation for maximal power helped identification of responsible mechanism and training adaptation [5].

Maximal power can be developed by increasing skills to develop high level of force in given speed and/ or showing given force at higher speed [6]. Isoinertial strength training reacts produced concentric force with constant eccentric resistance and enables development on unit power and explosive power by enabling contraction at muscle elongation phase. In optimally overloaded strength training, half squat and squat jump (SJ) are important movements to prevent force and speed decreases

(c) Kale M., Özmusul A., Heper E., 2019

doi:10.15561/18189172.2019.0204
[7]. Wisløff et al. [8] stated that amount of weight in a maximum half squat is highly related with acceleration and vertical jump (VJ). Additionally, $\mathrm{VJ}$ is generally used for evaluation isoinertial leg power since it includes simple and explosive movements [6].

$\mathrm{VJ}$ and horizontal jump (HJ), including sequences of complex movements, are related with leg muscle strength, explosive power, flexibility of muscles in vertical or horizontal jump as well as vertical and horizontal jump techniques. To provide an effective $\mathrm{VJ}$ or HJ, during application of the jump technique, joints should be flexion in proper angles. Maximal muscle contraction during extension and explosive application of the movement increases efficiency of VJ and $\mathrm{HJ}$ [9]. Height performed in $\mathrm{VJ}$ and distance performed in $\mathrm{HJ}$ evaluates leg power and these are closely related with speed performance [10]. Maulder et al. [11] found statistically significant positive relationships among horizontal force (HF), vertical force (VF), and sprint velocity. Different jump tests are applied to test different strenght qualities. Squat jump (SJ) evaluates leg explosive power under concentric conditions while countermovement jump (CMJ) measures leg power under slow stretch shortening cycle and low stretching overload conditions [12]. Due to stretch shortening cycle organised by high level of active movement during eccentric phase of CMJ stated by Cormie et al. [13], the most general dynamic performance test for neuromuscular fatigue during isoinertial resistance load is CMJ test [14]. $\mathrm{HJ}$ requires both horizontal and vertical propulsive force 
and this enables analysing activities that contain linear movements in horizontal plane [15]. However, there are limited number of studies to determine horizontal functional performance relationships. Nagona et al. [16] determined that during an active movement in standing broad jump (SBJ) by moving centre of mass to forward effectively, there were more effective mechanical energy transfer and high level muscle activations based on hip flexion of muscles such as rectus femoris. Therefore, such plyometric jumps that can be considered as elastic power with maximal strength and indicates high relationships with muscle fiber type, contraction speed, metabolic properties of muscle, neuromuscular activation, and kinetic energy transfer.

Sprint and jump require eccentric strength plays an important role to develop performance [17]. Eccentric strength training with isoinertial resistance training system will increase power by enabling force and speed as the muscle activitation [3] and compared to traditional weight training it provides higher muscle activation [4]. This supports that isoinertial resistance training will be a more effective training for development of $\mathrm{HF}$ and $\mathrm{VF}$ during sprint, $\mathrm{VJ}$ height performed during $\mathrm{SJ}$ and $\mathrm{CMJ}$, HJ distance performed during standing broad jump (SBJ) and standing broad countermovement jump (SBCMJ). Thus, the purpose of the currrent study was to investigate acute effects of isoinertial resistance application on sprint, $\mathrm{VJ}$, and $\mathrm{HJ}$ performance.

\section{Materials and methods}

\section{Participants}

Thirty two male volunteer Faculty of Sports Sciences students, being physically active at easy-medium level (weekly $\leq 3$ session aerobic activity), participated in this study. They were randomly divided into 2 groups as experimental group [(EG) $(\mathrm{n}=16$; age: $19.6 \pm 1.3$ years; height: $174.6 \pm 7.2 \mathrm{~cm}$; weight: $70.4 \pm 8.1 \mathrm{~kg}$ )] and control group $[(\mathrm{CG})(\mathrm{n}=16$; age: $20.5 \pm 2.1$ years; height: $178.1 \pm 5.5$; weight: $71.9 \pm 5.7 \mathrm{~kg})]$. The study was approved by Ethical Board of Anadolu University. Written informed consents were obtained from the subjects in accordance with the principle of Helsinki Declaration.

\section{Procedures}

Subjects participated in tests and squat workouts for trial and familiarization between the hours of 9.30 and 11.00 one week before the study. Additionally, EG participated in squat workouts with isoinertial resistance application. After 20min warm-up consisting of light tempo running, flexibility, and calistenic exercises, $2 \times 30 \mathrm{~m}$ sprints with 3-min interval were tested on non-motorised treadmill. Based on both leg (BL), dominant leg (DL), and non-dominant leg (NDL) that sprint parameters stride frequency (SF), stride length (SL), HF, VF, work (W) and power $(\mathrm{P})$ were determined. As stated by Kaçoglu and Kale [18], VJ and HJ tests were applied after $90 \mathrm{sec}$ passive rest. Pre-tests were completed 2 reps SJ, CMJ based on BL, DL, and NDL, SBJ and SBCMJ based on BL with 30sec interval between reps and 60sec passive rest between tests. Statistically better result of 2 trials for each jump were evaluated. After three days of break, before post-test, both groups applied 4-RM half squat workout with BL and $3 \mathrm{~min}$ passive rest was applied at the end. EG applied the workout with isoinertial resistance with isoinertial strength training system. After passive rest, tests were repeated with same protocols.

\section{Isoinersial Resistance Application Program}

While the subject was straight and parallel to wall, his elbows were bent $90^{\circ}$, hands on waist, eyes looking forward, femur of each leg parallel to ground, and $90^{\circ}$ knee angle, with isoinertial training system (Desmotec D11-V12 Version Sport Pro, Italy) linked to his waist with an adjustable rope, subjects applied 4-repetition maximal (4 RM) half squat workout. Power values from isoinertial resistance application are shown in Table 1.

Table 1. Power values in isoinersial resistance application of EG (ORT $\pm S S)$

\section{CONCENTRIC POWER (W) ECCENTRIC POWER (W)}

$551 \pm 290$ $507 \pm 246$

\section{Sprint Test}

Subjects were participated to $2 \times 30 \mathrm{~m}$ sprint test with 3 min intervals on non-motorised treadmill (Woodway Force 3.0, Woodway Inc., USA) after 20 min warmup consisting of light tempo running, flexibility, and calistenic exercises. Before testing, HF strain gauge was adjusted parallel to treadmill based on the waist level. Thirty meter sprint speed was calculated with $\left(\mathrm{V}_{\text {mean }}\right)=$ distance $(d) /$ time $(t)$ in terms of $\mathrm{m} . \mathrm{sn}^{-1}$. During $30 \mathrm{~m}$ sprint, mean $\mathrm{HF}\left(\mathrm{HF}_{\text {mean }}\right)$ and mean $\mathrm{VF}\left(\mathrm{VF}_{\text {mean }}\right)$ data were recorded to computer at $200 \mathrm{~Hz}$. The best $30 \mathrm{~m}$ sprint result was statistically analysed. $\mathrm{HF}_{\text {mean }}$ was calculated with $30 \mathrm{~m}$ sprint total $\mathrm{HF} / 30 \mathrm{~m}$ sprint total stride number formula in terms of Newton. $\mathrm{VF}_{\text {mean }}$ was calculated with 30 $\mathrm{m}$ sprint total $\mathrm{VF} / 30 \mathrm{~m}$ sprint total stride number formula in terms of Newton. Same calculation method was used for all other sprint parameters of both legs that are mean $\mathrm{SF}\left(\mathrm{SF}_{\text {mean }}\right)$, mean SL $\left(\mathrm{SL}_{\text {mean }}\right)$, mean $\mathrm{W}\left(\mathrm{W}_{\text {mean }}\right)$ and mean $\mathrm{P}\left(\mathrm{P}_{\text {mean }}\right)$, and also DL and NDL that are dominant leg $\mathrm{SF}\left(\mathrm{SF}_{\mathrm{DL}}\right)$, non-dominant leg $\left(\mathrm{SF}_{\mathrm{NDL}}\right)$, dominant leg SL $\left(\mathrm{SL}_{\mathrm{DL}}\right)$, non-dominant leg $\mathrm{SL}\left(\mathrm{SL}_{\mathrm{NDL}}\right)$, dominant leg $\mathrm{HF}$ $\left(\mathrm{HF}_{\mathrm{DL}}\right)$, non-dominant leg $\mathrm{HF}\left(\mathrm{HF}_{\mathrm{NDL}}\right)$, dominant leg $\mathrm{VF}$ $\left(\mathrm{VF}_{\mathrm{DL}}\right)$, non-dominant leg $\mathrm{VF}\left(\mathrm{VF}_{\mathrm{NDL}}\right)$, dominant leg W $\left(\mathrm{W}_{\mathrm{DL}}\right)$, non-dominant leg $\mathrm{W}\left(\mathrm{W}_{\mathrm{NDL}}\right)$, dominant leg $\mathrm{P}\left(\mathrm{P}_{\mathrm{DL}}\right)$, non-dominant leg $\mathrm{P}\left(\mathrm{P}_{\mathrm{NDL}}\right)$.

Vertical Jumps

SJ and CMJ tests based on BL, DL, NDL [both leg SJ $\left(\mathrm{SJ}_{\mathrm{BL}}\right)$, dominant leg $\mathrm{SJ}\left(\mathrm{SJ}_{\mathrm{DL}}\right)$, non-dominant leg SJ $\left(\mathrm{SJ}_{\mathrm{NDL}}\right)$, both leg CMJ $\left(\mathrm{CMJ}_{\mathrm{BL}}\right)$, dominant leg CMJ $\left(\mathrm{CMJ}_{\mathrm{DL}}\right)$, non-dominant leg $\mathrm{CMJ}\left(\mathrm{CMJ}_{\mathrm{NDL}}\right)$,] were applied to participants as VJ. To transfer data of jumping mat acted as electronic on-off switch to $1000 \mathrm{~Hz}$ computer, ESC 2XXX Series Data Acquisition computer software (Tümer Elektronik Ltd, Ankara, Turkey) was used and flight time of SJ and CMJ in BL, DL and NDL were 
transferred to computer. After each VJ test, subjects rested for $60 \mathrm{sec}$. For each VJ test, 2 trials were conducted with $30 \mathrm{sec}$ interval and higher jump height was considered for statistical evaluation. Each jump height was calculated with the formula adopted by Bosco et al. [19]: $h=g \cdot t_{f}^{2} \cdot 8^{-}$ ${ }^{1} .100$ where $\mathrm{h}$ - is the jump height $(\mathrm{cm}), \mathrm{g}$ - is gravitational acceleration $\left(9.81 \mathrm{~m} . \mathrm{s}^{-2}\right.$.), $\mathrm{t}_{\mathrm{f}}$ is flight time $(\mathrm{s})$. SJ was applied as vertical jump where feet were at shoulder width, eyes are focused straight, hands on waist, and waiting stable squat position $\left(85-90^{\circ}\right.$ knee angle) for 2-3 sec while CMJ was applied on the same mat where feet were at shoulder width, eyes are focused straight, hands on waist, and squating in shortest time possible from the standing position to on knees $\left(85-90^{\circ}\right.$ knee angle).

Horizontal Jumps

SBJ and SBCMJ tests based on BL were conducted as HJ test. SBJ was applied as broad jump where feet were at shoulder width, eyes are focused straight, hands on waist, and waiting stable squat position $\left(85-90^{\circ}\right.$ knee angle) for 2-3 sec while SBCMJ was applied as broad jump where feet were at shoulder width, eyes are focused straight, hands on waist, and squating in shortest time possible from the standing position to on knees $\left(85-90^{\circ}\right.$ knee angle). Distance between toe tip before broad jump and heel after broad jump was measured with a steel meter (Stabila, Germany) and recorded as $\mathrm{cm}$. After each horizontal jump test, participants rested for 60 sec. For each horizontal jump test, 2 trials were conducted with 30 sec interval and the longer jump distance was considered for statistical evaluation.

\section{Statistical Analysis}

Data analysis was conducted with SPSS 22 (SPSS Inc., Chicago, IL, USA). After determining mean, standard deviation, and percentage change, intra-group pre-test and post-test percentage changes were determined. Intragroup pre- and post-test results compared with Wilcoxon $\mathrm{T}$ test, and inter-group pre- and post-test results compared with Mann Whitney U test. Significance level was selected as $\mathrm{p} \leq 0.05$.

\section{Results}

As seen from Table 2, there were no statistically significant difference for all intra-group pre- and post-test results of $\mathrm{VJ}$ and $\mathrm{HJ}$ parameters of EG and $\mathrm{CG}$. Except the post-test result of $\mathrm{CMJ}_{\mathrm{NDL}}(\mathrm{Z}=-2.13 ; \mathrm{p}<0.05)$, there were no statistically significant differences for inter-group results of $\mathrm{VJ}$ and $\mathrm{HJ}$ parameters. When intra-group preand post-test $\%$ percentage was analysed, it was shown that $\mathrm{VJ}$ and $\mathrm{HJ}$ parameters were changed in between -9.3 and $-0.5 \%$ for EG, between -6.6 and $0.5 \%$ for CG.

As seen from Table 3, there were no statistically

Table 2. Pre- and post-test results of vertical and horizontal jump parameters

\begin{tabular}{|c|c|c|c|c|c|c|}
\hline JUMP PARAMETERS & & PRE-TEST & POST-TEST & $\%$ CHANGE & $\mathbf{Z}$ & $\mathbf{p}$ \\
\hline \multirow{4}{*}{$\mathrm{SJ}_{\mathrm{BL}}(\mathrm{cm})$} & EG & $34.9 \pm 4.7$ & $33.4 \pm 3.3$ & -4.2 & -0.75 & 0.45 \\
\hline & CG & $35.2 \pm 4.6$ & $34.4 \pm 4.1$ & -2.2 & -0.49 & 0.62 \\
\hline & $\mathbf{Z}$ & -0.28 & -0.53 & & & \\
\hline & p & 0.77 & 0.59 & & & \\
\hline \multirow{4}{*}{$\mathrm{SJ}_{\mathrm{DL}}(\mathrm{cm})$} & EG & $18.9 \pm 3.3$ & $17.8 \pm 2.6$ & -5.8 & -0.95 & 0.34 \\
\hline & CG & $20.3 \pm 3.6$ & $18.9 \pm 3.5$ & -6.6 & -1.32 & 0.17 \\
\hline & $\mathbf{Z}$ & -1.19 & -0.97 & & & \\
\hline & p & 0.23 & 0.33 & & & \\
\hline \multirow{4}{*}{$\mathrm{SJ}_{\mathrm{NDL}}(\mathrm{cm})$} & EG & $16.8 \pm 3.1$ & $15.5 \pm 2.2$ & -7.7 & -1.43 & 0.15 \\
\hline & CG & $17.7 \pm 2.9$ & $17.0 \pm 3.4$ & -3.9 & -0.60 & 0.55 \\
\hline & $\mathbf{Z}$ & -1.05 & -1.19 & & & \\
\hline & p & 0.29 & 0.23 & & & \\
\hline \multirow{4}{*}{$\mathrm{CMJ}_{\mathrm{BL}}(\mathrm{cm})$} & EG & $38.3 \pm 5.6$ & $36.6 \pm 3.9$ & -4.4 & -0.40 & 0.69 \\
\hline & CG & $38.4 \pm 3.9$ & $37.8 \pm 3.8$ & -1.6 & -0.52 & 0.60 \\
\hline & $\mathbf{Z}$ & -0.57 & -1.12 & & & \\
\hline & p & 0.57 & 0.26 & & & \\
\hline \multirow{4}{*}{$\mathrm{CMJ}_{\mathrm{DL}}(\mathrm{cm})$} & EG & $20.3 \pm 3.4$ & $19.2 \pm 3.2$ & -5.4 & -0.66 & 0.51 \\
\hline & CG & $20.9 \pm 4.1$ & $20.2 \pm 3.8$ & -3.4 & -0.49 & 0.63 \\
\hline & $\mathbf{Z}$ & -0.29 & -0.76 & & & \\
\hline & p & 0.78 & 0.45 & & & \\
\hline \multirow{4}{*}{$\mathrm{CMJ}_{\mathrm{NDL}}(\mathrm{cm})$} & EG & $18.2 \pm 3.8$ & $16.5 \pm 2.4$ & -9.3 & -1.40 & 0.16 \\
\hline & CG & $18.7 \pm 2.6$ & $18.8 \pm 3.5$ & 0.5 & -0.14 & 0.88 \\
\hline & $\mathbf{Z}$ & -0.47 & -2.13 & & & \\
\hline & p & 0.64 & $0.03 *$ & & & \\
\hline \multirow{4}{*}{ SBJ (m) } & EG & $1.82 \pm 0.16$ & $1.76 \pm 0.09$ & -3.2 & -0.60 & 0.55 \\
\hline & CG & $1.73 \pm 0.22$ & $1.70 \pm 0.19$ & -1.7 & -0.44 & 0.66 \\
\hline & $\mathbf{Z}$ & -1.85 & -1.77 & & & \\
\hline & p & 0.06 & 0.08 & & & \\
\hline \multirow{4}{*}{$\operatorname{SBCMJ}(\mathrm{m})$} & EG & $1.89 \pm 0.17$ & $1.88 \pm 0.11$ & -0.5 & -0.73 & 0.47 \\
\hline & CG & $1.84 \pm 0.26$ & $1.85 \pm 0.22$ & 0.5 & -0.31 & 0.76 \\
\hline & $\mathbf{Z}$ & -1.38 & -0.66 & & & \\
\hline & $p$ & 0.17 & 0.51 & & & \\
\hline
\end{tabular}

\footnotetext{
* Statistical significant pre-test inter-group difference, $p<0.05$.
} 
significant differences between intra-group pre- and posttest $\mathrm{V}_{\text {mean }}, \mathrm{SF}_{\text {mean }}, \mathrm{SL}_{\text {mean }}, \mathrm{HF}_{\text {mean }}, \mathrm{VF}_{\text {mean }}, \mathrm{W}_{\text {mean }}$ and $\mathrm{P}_{\text {mean }}$ results of BL sprint parameters. There was a statistically significant difference for inter-group pre-test results of $\mathrm{SL}_{\text {mean }}(\mathrm{Z}=-2.27 ; \mathrm{p}<0.02)$. There were no statistically significant inter-group differences in pre- and post-test $\mathrm{V}_{\text {mean }}, \mathrm{SF}_{\text {mean }}, \mathrm{HF}_{\text {mean }}, \mathrm{VF}_{\text {mean }}, \mathrm{W}_{\text {mean }}$ ve $\mathrm{P}_{\text {mean }}$ results of $\mathrm{BL}$ sprint parameters. In $\mathrm{EG}$, except $\mathrm{VF}_{\text {mean }}(2.1 \%)$, all other BL sprint parameters showed negative change between -1.2 and $-2.7 \%$. In $\mathrm{CG}$, except $\mathrm{HF}_{\text {mean }}$ and $\mathrm{VF}_{\text {mean }}(-0.5 \%$ and $-1.5 \%$ respectively), all other BL sprint parameters showed positive change between 0.7 and $1.9 \%$.

As seen from Table 4, there were no statistically significant intragroup differences between pre- and posttest DL and NDL sprint parameters of EC and GC, except statistically significant difference for $\mathrm{VF}_{\mathrm{DL}}$ parameter $(Z=-2.07 ; p<0.05)$ of $C G$. In terms of inter-group pre- and post-test $\mathrm{SL}_{\mathrm{NDL}}$ results, there were statistically significant differences (for pre-test $\mathrm{Z}=-1.99$ and for post-test $\mathrm{Z}=$ $2.28 ; p<0.05$ ) but there were no statistically significant differences for $\mathrm{SL}_{\mathrm{DL}}$ parameter. For $\mathrm{W}_{\mathrm{NDL}}$ results, there was only statistically significant inter-group pre-test difference (for pre-test $Z=-2.22 ; p<0.03$ ). Among EG preand post-test DL and NDL results, the highest changes were in $\mathrm{W}_{\mathrm{DL}}$ parameter with $6.6 \%$ positive change and in $\mathrm{W}_{\mathrm{NDL}}$ parameter with $-6.6 \%$ negative change. Among CG pre- and post-test DL and NDL results, the highest changes were in $\mathrm{W}_{\mathrm{NDL}}$ parameter with $2.8 \%$ positive change and in $\mathrm{VF}_{\mathrm{DL}}$ parameter with $-6.6 \%$ negative change.

\section{Discussion}

In this study, all $\mathrm{VJ}$ and $\mathrm{HJ}$ parameters of $\mathrm{EG}$ and $\mathrm{CG}$ which were $\mathrm{SJ}_{\mathrm{BL}}, \mathrm{SJ}_{\mathrm{DL}}, \mathrm{SJ}_{\mathrm{NDL}}, \mathrm{CMJ}_{\mathrm{BL}}, \mathrm{CMJ}_{\mathrm{DL}}, \mathrm{CMJ}_{\mathrm{NDL}}$, SBJ and SBCMJ showed no statistically significant differences for intra-group pre- and post-test results. However, when percentage changes were analysed, there were decreasing trends in all pre- and post-test results of EG. For VJ, Howatson et al. [20] found that $\mathrm{CMJ}_{\mathrm{BL}}$ was unchanged one day after isoinertial load application. Kennedy and Drake [21] determined that right after isoinertial resistance aplication, $\mathrm{CMJ}_{\mathrm{BL}}$ statistically decreased $(\mathrm{p}<0.05)(36.1 \pm 0.78 \mathrm{~cm}$ vs. $32.4 \pm 0.74 \mathrm{~cm})$. In current study, there was no statistically significant difference, however, $\mathrm{CMJ}_{\mathrm{BL}}$ decreased from $38.3 \pm 5.6 \mathrm{~cm}$ to $36.6 \pm 3.9 \mathrm{~cm}$. The jump test $3 \mathrm{~min}$ after isoinertial power application may be a factor causing such a difference. Sequence of motor unit recruitment in voluntary muscle contraction is from small motor unit to large motor unit based on intensity of stimulus and sub-maximal voluntary muscle contraction only recruites small motor units of muscle. During an eccentric contraction that contains maximal or supra-maximal contractions that cause recruitment of large motor units, elastic energy is stored in serial elastic elements and is used during concentric contraction where contractile elements and serial elastic elements shorten [22]. For the activation that has a concentric contraction during $\mathrm{SJ}$, more time is required for activation in $\mathrm{CMJ}$ that contain a concentric contraction right after an eccentric contraction [23]. Therefore, with

Table 3. Pre- and post-test results of $30 \mathrm{~m} \mathrm{BL}$ sprint parameters

\begin{tabular}{|c|c|c|c|c|c|c|}
\hline \multicolumn{2}{|c|}{ BL SPRINT PARAMETERS } & \multirow{2}{*}{$\begin{array}{l}\text { PRE-TEST } \\
4.69 \pm 0.29\end{array}$} & \multirow{2}{*}{$\begin{array}{l}\text { POST-TEST } \\
4.62 \pm 0.36\end{array}$} & \multirow{2}{*}{$\begin{array}{l}\text { \% CHANGE } \\
-1.5\end{array}$} & \multirow{2}{*}{\begin{tabular}{|l|}
$\mathbf{Z}$ \\
-0.21
\end{tabular}} & \multirow{2}{*}{$\begin{array}{l}\mathbf{p} \\
0.84\end{array}$} \\
\hline \multirow{4}{*}{$V_{\text {mean }}\left(m \cdot s n^{-1}\right)$} & EG & & & & & \\
\hline & CG & $4.54 \pm 0.27$ & $4.57 \pm 0.34$ & 0.7 & -0.31 & 0.76 \\
\hline & $\mathbf{Z}$ & -1.69 & -1.02 & & & \\
\hline & p & 0.90 & 0.31 & & & \\
\hline \multirow{4}{*}{$S F_{\text {mean }}(H z)$} & EG & $4.06 \pm 0.28$ & $4.01 \pm 0.30$ & -1.2 & -0.57 & 0.57 \\
\hline & CG & $4.17 \pm 0.26$ & $4.22 \pm 0.33$ & 1.2 & -0.26 & 0.79 \\
\hline & $\mathbf{Z}$ & -1.45 & -1.69 & & & \\
\hline & p & 0.15 & 0.09 & & & \\
\hline \multirow{4}{*}{$S L_{\text {mean }}(m)$} & EG & $1.50 \pm 0.19$ & $1.48 \pm 0.27$ & -1.3 & -0.96 & 0.34 \\
\hline & CG & $1.33 \pm 0.23$ & $1.35 \pm 0.22$ & 1.5 & -0.18 & 0.86 \\
\hline & Z & -2.27 & -1.59 & & & \\
\hline & p & $0.02 *$ & 0.11 & & & \\
\hline \multirow{4}{*}{$\mathrm{HF}_{\text {mean }}(\mathrm{N})$} & EG & $145 \pm 11$ & $143 \pm 13$ & -1.3 & -0.31 & 0.76 \\
\hline & CG & $146 \pm 11$ & $145 \pm 12$ & -0.5 & -0.46 & 0.64 \\
\hline & $\mathbf{Z}$ & -0.39 & -0.38 & & & \\
\hline & p & 0.69 & 0.71 & & & \\
\hline \multirow{4}{*}{$V F_{\text {mean }}(N)$} & EG & $643 \pm 73$ & $657 \pm 72$ & 2.1 & -0.31 & 0.76 \\
\hline & CG & $655 \pm 51$ & $645 \pm 50$ & -1.5 & -0.62 & 0.54 \\
\hline & Z & -0.26 & -0.53 & & & \\
\hline & p & 0.79 & 0.60 & & & \\
\hline \multirow{4}{*}{$W_{\text {mean }}(J)$} & EG & $218 \pm 33$ & $215 \pm 54$ & -1.2 & -0.41 & 0.68 \\
\hline & CG & $196 \pm 42$ & $198 \pm 44$ & 1.4 & -0.05 & 0.96 \\
\hline & Z & -1.53 & -1.05 & & & \\
\hline & p & 0.13 & 0.29 & & & \\
\hline \multirow{4}{*}{$P_{\text {mean }}(W)$} & EG & $701 \pm 91$ & $682 \pm 92$ & -2.7 & -0.21 & 0.84 \\
\hline & CG & $677 \pm 90$ & $689 \pm 95$ & 1.9 & -0.10 & 0.92 \\
\hline & $Z$ & -0.72 & -0.11 & & & \\
\hline & $p$ & 0.47 & 0.91 & & & \\
\hline
\end{tabular}

* Statistical significant pre-test inter-group difference, $p<0.05$. 
Table 4. Pre- and post-test results of $30 \mathrm{~m} \mathrm{DL}$ and NDL sprint parameters

\begin{tabular}{|c|c|c|c|c|c|c|}
\hline \multicolumn{2}{|c|}{ DL and NDL SPRINT PARAMETERS } & PRE-TEST & POST-TEST & \% CHANGE & $\mathbf{Z}$ & $\mathbf{p}$ \\
\hline \multirow{4}{*}{$\mathrm{SF}_{\mathrm{DL}}(\mathrm{Hz})$} & EG & $3.88 \pm 0.33$ & $3.88 \pm 0.34$ & 0.0 & -0.10 & 0.92 \\
\hline & CG & $3.94 \pm 0.36$ & $4.00 \pm 0.39$ & 1.5 & -0.62 & 0.54 \\
\hline & $\mathbf{Z}$ & -0.69 & -0.77 & & & \\
\hline & $\mathbf{p}$ & 0.49 & 0.44 & & & \\
\hline \multirow{4}{*}{$S F_{N D L}(H z)$} & EG & $4.24 \pm 0.34$ & $4.14 \pm 0.34$ & -2.3 & -0.72 & 0.47 \\
\hline & CG & $4.39 \pm 0.36$ & $4.43 \pm 0.39$ & 0.9 & -0.36 & 0.72 \\
\hline & Z & -1.24 & -1.87 & & & \\
\hline & p & 0.21 & 0.06 & & & \\
\hline \multirow{4}{*}{$S L_{D L}(m)$} & EG & $1.61 \pm 0.24$ & $1.57 \pm 0.32$ & -2.4 & -0.91 & 0.37 \\
\hline & CG & $1.48 \pm 0.34$ & $1.46 \pm 0.29$ & -1.4 & -0.41 & 0.68 \\
\hline & $Z$ & -1.69 & -1.09 & & & \\
\hline & p & 0.09 & 0.27 & & & \\
\hline \multirow{4}{*}{$S L_{N D L}(m)$} & EG & $1.28 \pm 0.19$ & $1.32 \pm 0.23$ & 3.1 & -0.43 & 0.67 \\
\hline & CG & $1.16 \pm 0.18$ & $1.17 \pm 0.17$ & 0.9 & -0.23 & 0.82 \\
\hline & $Z$ & -1.99 & -2.28 & & & \\
\hline & $p$ & $0.05^{*}$ & $0.02 *$ & & & \\
\hline \multirow{4}{*}{$\mathrm{HF}_{\mathrm{DL}}(\mathrm{N})$} & EG & $141 \pm 13$ & $139 \pm 18$ & -1.4 & -0.57 & 0.57 \\
\hline & CG & $141 \pm 16$ & $143 \pm 12$ & 1.4 & -0.31 & 0.76 \\
\hline & Z & -0.08 & -0.91 & & & \\
\hline & $p$ & 0.94 & 0.37 & & & \\
\hline \multirow{4}{*}{$\mathrm{HF}_{\mathrm{NDL}}(\mathrm{N})$} & EG & $148 \pm 19$ & $146 \pm 16$ & -1.3 & -0.47 & 0.64 \\
\hline & CG & $150 \pm 13$ & $146 \pm 18$ & -2.7 & -0.93 & 0.35 \\
\hline & Z & -0.53 & -0.26 & & & \\
\hline & p & 0.60 & 0.79 & & & \\
\hline \multirow{4}{*}{$\mathrm{VF}_{\mathrm{DL}}(\mathrm{N})$} & EG & $647 \pm 83$ & $662 \pm 95$ & 2.3 & -0.78 & 0.44 \\
\hline & CG & $675 \pm 47$ & $634 \pm 67$ & -6.1 & -2.07 & $0.04^{\text {a }}$ \\
\hline & Z & -1.28 & -0.72 & & & \\
\hline & $p$ & 0.20 & 0.47 & & & \\
\hline \multirow{4}{*}{$V F_{N D L}(N)$} & EG & $639 \pm 76$ & $650 \pm 79$ & 1.7 & -0.16 & 0.88 \\
\hline & CG & $633 \pm 48$ & $648 \pm 77$ & 2.4 & -0.62 & 0.54 \\
\hline & Z & 0.01 & -0.12 & & & \\
\hline & $p$ & 1.00 & 0.91 & & & \\
\hline \multirow{4}{*}{$W_{D L}(J)$} & EG & $181 \pm 40$ & $193 \pm 46$ & 6.6 & -0.93 & 0.35 \\
\hline & CG & $179 \pm 43$ & $178 \pm 41$ & -0.6 & -0.36 & 0.72 \\
\hline & Z & -0.57 & -1.21 & & & \\
\hline & $p$ & 0.57 & 0.23 & & & \\
\hline \multirow{4}{*}{$W_{N D L}(J)$} & EG & $254 \pm 48$ & $237 \pm 74$ & -6.6 & -1.14 & 0.26 \\
\hline & CG & $212 \pm 49$ & $218 \pm 58$ & 2.8 & -0.16 & 0.87 \\
\hline & $Z$ & -2.22 & -0.68 & & & \\
\hline & $p$ & $0.03^{*}$ & 0.49 & & & \\
\hline \multirow{4}{*}{$P_{D L}(W)$} & EG & $693 \pm 107$ & $675 \pm 110$ & -2.5 & -0.36 & 0.72 \\
\hline & CG & $674 \pm 106$ & $687 \pm 94$ & 1.9 & -0.16 & 0.87 \\
\hline & Z & -0.45 & -0.26 & & & \\
\hline & p & 0.65 & 0.79 & & & \\
\hline \multirow{4}{*}{$P_{N D L}(W)$} & EG & $709 \pm 110$ & $689 \pm 97$ & -2.8 & -0.52 & 0.61 \\
\hline & CG & $678 \pm 97$ & $691 \pm 126$ & 1.9 & -0.31 & 0.76 \\
\hline & $\mathbf{Z}$ & -1.09 & -0.42 & & & \\
\hline & $p$ & 0.27 & 0.68 & & & \\
\hline
\end{tabular}

* Statistical significant pre-test and post-test inter-group differences, $\mathrm{p}<0.05$; ${ }^{\square}$ Statistical significant pre-test and posttest intra-group difference, $\mathrm{p}<0.05$.

preliminary overload effect in $\mathrm{CMJ}$, eccentric contraction caused an increase muscle force at the beginning of concentric contraction [24]. Jakobsen et al. [17] showed that eccentric force training of hamstrings muscle group, recruited large motor units, positively increased eccentric force related performance development such as $\mathrm{VJ}$ and HJ. Nosaka et al. [25] stated that force production of an eccentric contraction is 5 times more than force producted of a concentric contraction. Vazquez-Guerreo and Moras [26] determined that since force of resistance in isoinertial system is dynamic, there is same or more force development than same movements with traditional weight training. Norrbrand et al. [4] stated that eccentric force training with isoinertial resistance training system provided more muscle activation on eccentric muscle contraction compared to traditional weight training. In current study, except the post-test result of $\mathrm{CMJ}_{\mathrm{NDL}}$ parameter $(Z=-2.13 ; p<0.05)$, there were no statistically significant differences in all other $\mathrm{VJ}$ and $\mathrm{HJ}$ parameters. This significant difference shows that $4 \mathrm{RM}$ isoinertial resistance application had acute effect on NDL. Based on this result, it could be thought that isoinertial resistance 
training for $\mathrm{CMJ}_{\mathrm{NDL}}$ can benefit to development of NDL elastic power.

In this study, there were no statistically significant differences between intragroup pre- and post-test results of $\mathrm{EG}$ and $\mathrm{CG}$ for $\mathrm{V}_{\text {mean }}, \mathrm{SF}_{\text {mean }}, \mathrm{SL}_{\text {mean }}, \mathrm{HF}_{\text {mean, }}, \mathrm{VF}_{\text {mean }}$, $\mathrm{W}_{\text {mean }}$, and $\mathrm{P}_{\text {mean }}$. These results indicate that $4 \mathrm{RM}$ isoinertial resistance applicaiton had no acute effect on all sprint parameters but statistically significant intergroup pre-test difference $(\mathrm{Z}=-2.27 ; \mathrm{p}<0.02)$ and no statistically significant post-test difference for $\mathrm{SL}_{\text {mean }}$ indicated that in EG, 4 RM isoinertial resistance training had an acute effect. Accordingly, when SL was analysed for DL and NDL, intra-group and inter-group pre- and post-test results were compared for $\mathrm{SL}_{\mathrm{DL}}$, there were no statistically significant differences. However, there were statistically significant intergroup differences for pre- and post-test results of $\mathrm{SL}_{\mathrm{NDL}}(\mathrm{Z}=-1.99$ and $-2.28 ; \mathrm{p}<0.05$, respectively). Therefore, this shows that $4 \mathrm{RM}$ isoinertial resistance application acutely effected $\mathrm{SL}_{\mathrm{NDL}}$. Pre- and post-test results of EG, decreased $\mathrm{SL}_{\mathrm{DL}}$ (from $1.61 \pm 0.24 \mathrm{~m}$ to $1.57 \pm 0.32 \mathrm{~m}$ ) and increased $\mathrm{SL}_{\mathrm{NDL}}$ (from $1.28 \pm 0.19 \mathrm{~m}$ to $1.32 \pm 0.23 \mathrm{~m}$ ), indicated that this could help tolerating bilateral leg imbalance. When inter-group results were compared, while there was statistically significant pretest difference for $\mathrm{W}_{\mathrm{NDL}}(\mathrm{Z}=-2.22 ; \mathrm{p}<0.03)$, no statistically significant difference for post-test. In addition, increased $\mathrm{W}_{\mathrm{DL}}$ (from $181 \pm 40 \mathrm{~J}$ to $193 \pm 46 \mathrm{~J}$ ) and decreased $\mathrm{W}_{\mathrm{NDL}}$ (from $254 \pm 48 \mathrm{~J}$ to $237 \pm 74 \mathrm{~J}$ ) in pre-test and post-test of
EG supports this idea. As stated by Chu and Korchemny [27], muscles are intensively contracted, relaxed, and strecthed during ballistic cyclic movements of sprint that absorb shocks and amortise external factors by moving body segments in different forms and range of motion accelerate or decelerate to balance joints and determine the best force application. Based on this force, SF and SL parameters of both legs are at optimum level. Therefore, while lower SF and higher SL was displayed in DL, higher SF and lower SL was displayed in NDL. Based on this, it is believed that high differences in SF, HF, VF, $\mathrm{W}, \mathrm{P}$, and especially SL could be eliminated or minimised in individuals with isoinertial resistance training based on $30 \mathrm{~m}$ sprint in DL and NDL.

As a result of the effect of acute isoinertial resistance application to SL especially to $\mathrm{SL}_{\mathrm{NDL}}$, it is concluded that long-term isoinertial resistance training can support sprint performance with tolerating SL imbalance between DL and NDL. In addition, statistically significant difference for intergroup $\mathrm{CMJ}_{\mathrm{NDL}}$ showed that isoinertial resistance application for $\mathrm{CMJ}_{\mathrm{NDL}}$ can develop NDL elastic power.

\section{Acknowledgements}

This study was supported by Anadolu University Scientific Research Projects by Project 1706S376.

\section{Conflicts of Interest}

The authors declare no conflicts of interest.

\section{References}

1. Loturco I, Ugrinowitsch C, Roschel H, Lopes Mellinger A, Gomes F, Tricoli V, et al. Distinct temporal organizations of the strength- and power-training loads produce similar performance improvements. Journal of Strength and Conditioning Research, 2013;27(1):188- 194. https://doi.org/10.1519/JSC.0b013e3182503807

2. Aboodarda SJ, Page PA, Behm DG. Muscle activation comparisons between elastic and isoinertial resistance: A meta-analysis. Clinical Biomechanics, 2016; 39:52- 61. https://doi.org/10.1016/j.clinbiomech.2016.09.008

3. Kellett M, Patroni P, Calvetti M, Susta D. Enhanced power after a 4-week submaximal isoinertial training: a pilot study. Proceedings of the 2014 NSCA National Strength and Conditioning Association International Conference; 2014 June 26-28; Murcia, Spain: CCD, 25;10(9):S188.

4. Norrbrand L, Pozzo M, Tesch PA. Flywheel resistance training calls for greater eccentric muscle activation than weight training. European Journal of Applied Physiology, 2010;110(5):997- 1005. https://doi.org/10.1007/s00421-010-1575-7

5. Samozino P, Rejc E, Di Prampero PE, Belli A, Morin JB. Optimal force-velocity profile in ballistic movements - altius: Citius or fortius? Medicine and Science in Sports and Exercise, 2012;44(2):313- 322. https://doi.org/10.1249/MSS.0b013e31822d757a

6. Cormie P, McGuigan MR, Newton RU. Developing maximal neuromuscular power. Part 2. Training considerations for improving maximal power production. Sports Medicine, 2011;41(2):125- 146. https://doi.org/10.2165/11538500-000000000-00000

7. Loturco I, Pereira LA, Kobal R, Maldonado T, Piazzi
$\mathrm{AF}$, Bottino A, et al. Improving sprint performance in soccer: Effectiveness of jump squat and Olympic push press exercises. PloS One, 2016;11(4):e0153958 . https://doi.org/10.1371/journal.pone.0153958

8. Wisløff U, Castagna C, Helgerud J, Jones R, Hoff J. Strong correlation of maximal squat strength with sprint performance and vertical jump height in elite soccer players. British Journal of Sports Medicine, 2004;38(3):285- 288. https://doi.org/10.1136/bjsm.2002.002071

9. Bobbert MF, Gerritsen KG, Litjens MC, Van Soest AJ. Why is countermovement jump height greater than squat jump height? Medicine and Science in Sports and Exercise, 1996;28(12):1402- 1412. https://doi.org/10.1097/00005768-199611000-00009

10.Perez-Gomez J, Rodrigez GV, Ara I, Olmedillas H, Chavarren $\mathrm{J}$, Gonzalez-Henriquez JJ, et al. Role of muscle mass on sprint performance: gender differences? European Journal of Applied Physiology, 2008;102(6):685- 94. https://doi.org/10.1007/s00421-007-0648-8

11. Maulder P, Bradshaw E, Koegh J. Jump kinetic determinations of sprint acceleration performance from starting blocks in male sprinters. Journal of Sports Science and Medicine, 2006;5(2):359-366.

12.Plisk S, Beachle TR, Earle RW. Speed, agility, and speedendurance development. In: Beachle TR, Earle RW, editors. Essentials of Strength Training and Conditioning. Champaigh, IL: Human Kinetics; 2008. p. 458-485.

13. Cormie P, McGuigan MR, Newton RU. Adaptations in athletic performance after ballistic power versus strength training. Medicine and Science in Sports and Exercise, 2010;42(8):1582- 1598. https://doi.org/10.1249/MSS.0b013e3181d2013a 
14.Raeder C, Wiewelhove T, Westphal-Martinez MP, Fernandez J, de Paula Simola RA, Kellmann M, et al. Neuromuscular fatigue and physiological responses after five dynamic squat exercise protocols. Journal of Strength and Conditioning Research, 2016;30(4):953- 965. https://doi.org/10.1519/JSC.0000000000001181

15. Maulder P, Cronin J. Horizontal and vertical jump assesment: reliability, symmetry, discriminative, and predictive ability. Physical Therapy in Sport, 2005;6(2):74- 82. https://doi.org/10.1016/j.ptsp.2005.01.001

16.Nagona A, Komura T, Fukashiro S. Optimal coordination of maximal-effort horizontal and vertical jump motions-a computer simulation study. Biomedical Engineering Online, 2007;6(1):20 https://doi.org/10.1186/1475-925X-6-20

17.JakobsenMD, SundstrupE,AndersenCH,PerssonR,ZebisMK, Andersen LL. Effectiveness of hamstring knee rehabilitation exercise performed in training machine vs. elastic resistance: electromyography evaluation study. American Journal of Physical Medicine and Rehabilitaion, 2014;93(4):320- 327. https://doi.org/10.1097/PHM.0000000000000043

18.Kaçoglu C, Kale M. Acute effects of lower body electromyostimulation application with two different frequencies on isokinetic strength and jumping performance. Journal of Physical Education and Sport, 2016;16(1):38-45.

19.Bosco C, Luhtanen P, Komi PV. A simple method for measurement of mechanical power in jumping. European Journal of Applied Physiology, 1983;50(2):273- 282. https://doi.org/10.1007/BF00422166

20.Howatson G, Brandon R, Hunter AM. The response to and recovery from maximum-strength and -power training in elite track and field athletes. International Journal of Sports Physiology and Performance, 2016;11(3):356- 362. https://doi.org/10.1123/ijspp.2015-0235

21.Kennedy RA, Drake D. Dissociated time course of recovery between strength and power after isoinertial resistance loading in rugby union players. Journal of Strength and Conditioning Research, 2018;32(3):748-755.

22.Padulo J, Laffaye G, Ardigò LP, Chamari K. Concentric and eccentric: muscle contraction or exercise? Journal of Sports Science and Medicine, 2013;312(3):608-609.

23.Van Hooren B, Zolotarjova J. The difference between countermovement and squat jump performance: A review of underlying mechanisms with practical applications. Journal of Strength and Conditioning Research, 2017;31(7):2011- 2020. https://doi.org/10.1519/JSC.0000000000001913

24.Enoka RM. Neuromechanical Basis of Kinesiology, $2^{\text {nd }}$ Edition. Champaign, IL: Human Kinetics; 1996.

25.Nosaka K, Newton MJ, Sacco P. Attenuation of protective effect against eccentric exercise induced muscle damage. Canadian Journal of Applied Physiolgy, 2005;30(5):529- 542. https://doi.org/10.1139/h05-139

26.Vazquez-Guerreo J, Moras G. Changes in muscular architecture and execution velocity during squats performed using the versapulley under stable and unstable conditions in junior elite basketball players. Cuader-nos de Psicología del Deporte, 2015;15(3):243-252.

27.Chu D, Korchemny R. Sprinting stride actions. Analysis and evaluation. National Strength and Conditioning Association Journal, 1989;11(6):6-8, 81- 85. https://doi.org/10.1519/0744-0049(1989)011<0006:SSAAA $\mathrm{E}>2.3 . \mathrm{CO} ; 2$

\section{Information about the authors:}

Kale M.; (Corresponding author); http://orcid.org/0000-0002-1960-2234; mkale@eskisehir.edu.tr; Department of Coaching Education, Faculty of Sport Sciences, Eskişehir Technical University ; Eskişehir Teknik Üniversitesi 2 Eylül Kampüsü, 26555 Tepebaşı/Eskişehir, Turkey.

Özmusul A.; http://orcid.org/0000-0002-0294-2339; aozmusul434@gmail.com; Department of Physical Education and Sports, Graduate School of Health Sciences, Anadolu University ; Yeşiltepe Mahallesi, Yunus Emre Kampüsü, 26470 Tepebaşı/ Eskişehir, Turkey.

Heper E.; http:/orcid.org/0000-0002-3671-4393; eheper@eskisehir.edu.tr; Department of Coaching Education, Faculty of Sport Sciences, Eskişehir Technical University; Eskişehir Teknik Üniversitesi 2 Eylül Kampüsü, 26555 Tepebaşı/Eskişehir, Turkey.

Cite this article as:

Kale M, Özmusul A, Heper E. Acute effects of isoinertial resistance application on sprint, vertical and horizontal jump performance. Pedagogics, psychology, medical-biological problems of physical training and sports, 2019;23(2):76-82. https://doi.org/10.15561/18189172.2019.0204

The electronic version of this article is the complete one and can be found online at: https://www.sportpedagogy.org.ua/index.php/PPS/issue/archive

This is an Open Access article distributed under the terms of the Creative Commons Attribution License, which permits unrestricted use, distribution, and reproduction in any medium, provided the original work is properly cited (http://creativecommons.org/licenses/by/4.0/deed.en).

Received: 07.02.2019

Accepted: 12.03.2019; Published: 30.04.2019 